\title{
Efeito de doses de fósforo na produtividade e armazenamento pós-colheita de dois cultivares de cebola
}

\author{
Geraldo Milanez de Resende ${ }^{*}$, Nivaldo Duarte Costa ${ }^{2}$, Jony Eishi Yuri ${ }^{3}$ \\ 10.1590/0034-737X201663020017
}

\section{RESUMO}

O fósforo é, reconhecidamente, o nutriente-chave para a obtenção de produtividades elevadas e tem sido o macronutriente que mais frequentemente limita a produção, em solos pobres nesse nutriente. Com o objetivo de avaliar a produtividade e armazenamento pós-colheita de bulbos de cultivares de cebola, em função de doses de fósforo, conduziu-se um experimento, de maio a dezembro de 2011, em Petrolina-PE. O delineamento experimental utilizado foi de blocos ao acaso, no esquema fatorial $5 \times 2$, compreendendo cinco doses de fósforo $\left(0 ; 60 ; 120,180\right.$ e $\left.240 \mathrm{~kg} \mathrm{ha}^{-1}\right)$ e dois cultivares (Franciscana IPA-10 e ValeOuro IPA-11), com quatro repetições. Os cultivares Franciscana IPA-10 (74,6 tha-1) e Vale Ouro IPA-11 (76,1 t ha-1) não apresentaram diferenças significativas para produtividade comercial de bulbos. Maior produtividade foi obtida na dose de $132 \mathrm{~kg} \mathrm{ha}^{-1} \mathrm{de}_{2} \mathrm{O}_{5}$, associada à dose mais econômica de $130 \mathrm{~kg} \mathrm{ha}^{-1}$ de $\mathrm{P}_{2} \mathrm{O}_{5}$. Verificou-se com o aumento das doses uma redução gradativa da produção de bulbos considerados não comerciais (refugos), sendo a menor produção estimada de refugos a obtida com a dose de $124 \mathrm{~kg} \mathrm{ha}^{-1}$ de $\mathrm{P}_{2} \mathrm{O}_{5}$. Bulbos comerciais com maiores massa fresca e diâmetro foram obtidos com o incremento das doses. Não se verificou perda de massa significativa aos 30 dias após cura, para doses ou cultivares. Aos 60 dias após cura, detectou-se efeito significativo com menor perda para o cultivar Franciscana IPA-10 (27,2\%) em comparação com o ValeOuro IPA-11 (31,9\%).

Palavras-chave: Allium cepa L., rendimento, nutrição, conservação.

\section{ABSTRACT}

\section{Phosphorus levels on yield and postharvest storage of onion cultivars}

Phosphorus is recognized as a key nutrient to obtain high productivity and macronutrient that has been most often limits the production in soil poor in nutrient. With the objective of evaluating the influence of phosphorus levels and cultivars on yield and storage postharvest was carried one trial in Petrolina-PE, Brazil, from May to December 2011. The experimental design was a completely randomized block in a 5 x 2 factorial scheme, composed of five phosphorus levels $\left(0 ; 60 ; 120,180\right.$ and $\left.240 \mathrm{~kg} \mathrm{ha}^{-1}\right)$ and two cultivar (Franciscana IPA-10 e ValeOuro IPA-11) with four replications. The Franciscana IPA-10 cultivars (74.6 $\left.\mathrm{t} \mathrm{ha}^{-1}\right)$ and ValeOuro IPA-11 (76.1 $\left.\mathrm{t} \mathrm{ha}^{-1}\right)$ showed no significant differences in commercial yield of bulbs. Highest commercial yield were obtained at dose of $132 \mathrm{~kg} \mathrm{ha}^{-1} \mathrm{P}_{2} \mathrm{O}_{5}$ was associated with economical dose of $130 \mathrm{~kg} \mathrm{ha}^{-1} \mathrm{P}_{2} \mathrm{O}_{5}$. It was verified with increasing levels, a gradual reduction in the production of bulbs considered non-commercial (culls), and the lower production estimated of culls was estimated dose of $121 \mathrm{~kg}$ ha${ }^{1} \mathrm{P}_{2} \mathrm{O}_{5}$. Commercial bulbs of higher fresh mass and diameter were obtained with the increment of phosphorus. There was no loss of significant mass after 30 days cure at doses or cultivars. At 60 after curing, it was detected a significant effect only for cultivars with less loss to cultivar Franciscana IPA-10 (27.2\%) compared the cultivar ValeOuro IPA-11 $(31.9 \%)$.

Key words: Allium cepa L., yield, nutrition, conservation.

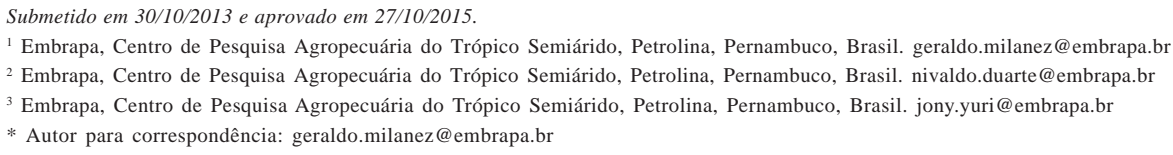




\section{INTRODUÇÃO}

No Brasil, a cebola (Allium cepa L.) é considerada a terceira hortaliça mais importante, perdendo para batata e tomate, sendo consumida in natura em saladas ou como condimento (Resende et al., 2002). Em 2012, a produtividade média nacional situou-se em torno de $24,7 \mathrm{t} \mathrm{ha}^{-1}$, sendo que, nos estados de Pernambuco e Bahia, maiores produtores do nordeste, alcançaram-se produtividades médias de 29,0 e de 20,4 $\mathrm{t} \mathrm{ha}^{-1}$, respectivamente (IBGE, 2013).

Embora o fósforo (P) seja acumulado em pequena quantidade por planta de cebola, de 31,23 a 33,35 mg (Vidigal et al., 2010; Porto et al., 2007), sua participação nos processos de absorção iônica, fotossíntese, respiração, sínteses, multiplicação e diferenciação celular e herança, denota que é um nutriente com expressiva ação na formação da produtividade e da qualidade do bulbo. Desse modo, limitações na disponibilidade de P, no início do ciclo vegetativo, podem resultar em restrições do desenvolvimento, das quais a planta não se recupera, posteriormente, mesmo aumentando-se o suprimento a níveis adequados. $\mathrm{O}$ suprimento adequado de P é essencial desde os estádios iniciais de crescimento da planta (Grant et al., 2001). O fósforo tem sido o macronutriente que mais frequentemente limita a produção, em condições de solos pobres em seu conteúdo (Tawaraya et al., 2012), havendo aparente contradição entre a pequena exigência da cultura e a resposta altamente positiva à adubação fosfatada (Filgueira, 2008). Como consequência, nas adubações, é o fósforo o nutriente que entra em maiores proporções (Coutinho et al., 1993). Estima-se que a produtividade das culturas é limitada pela deficiência de fósforo em 30 a $40 \%$ das terras agricultáveis do mundo (Vance et al., 2003; Balemi \& Negisho, 2012).

Pesquisas com fósforo, na cultura de cebola, no Brasil, são praticamente inexistentes, nas últimas duas décadas. Lima et al. (1984) e Wamser et al. (2011) observaram efeitos significativos na produtividade com as doses de 127,0 e de $60,0 \mathrm{~kg} \mathrm{ha}^{-1}$ de $\mathrm{P}_{2} \mathrm{O}_{5}$, respectivamente. Todavia, estudos internacionais sobre a capacidade de resposta dessa cultura a doses de fósforo, informam que o nutriente contribui para maior produtividade. A cebola respondeu significativamente à aplicação de fósforo em taxas entre 33 e $100 \mathrm{~kg} \mathrm{ha}^{-1}$ (Singh \& Singh, 2000; Meena et al., 2007; Yadav \& Prasad, 2009; Dudhat et al., 2010). Todavia, esses autores não apresentaram os resultados das análises de solo com os respectivos teores de nutrientes. Com $16 \mathrm{mg} \mathrm{dm}^{-3}$ de fósforo, em Vertissolo argiloso (alto teor do nutriente), Tekalign et al. (2012) não observaram efeito significativo na produtividade da cebola até doses de $40 \mathrm{~kg} \mathrm{ha}^{-1}$.
Cada vez mais os sistemas agrícolas buscam maior eficiência no uso dos recursos naturais disponíveis. $\mathrm{O}$ fósforo figura, nesse contexto, como elemento essencial à produção agrícola, sendo, porém finito e insubstituível (Malavolta, 2006) e com a longevidade de suas jazidas de, aproximadamente, 90 anos (Fixen, 2009). O estímulo às boas práticas para o uso correto dos fertilizantes, em geral, além de passar pelas consequências econômicas, também passa pelo manejo mais sustentável dos nutrientes. Isso considera que os nutrientes sejam escolhidos com a composição e combinação adequadas e que sejam aplicados na dose, época e locais corretos (Bruulsema et al., 2009).

O objetivo deste trabalho foi definir a dose adequada e economicamente viável de fósforo para melhores efeitos na produtividade e conservação, no armazenamento pós-colheita, de bulbos de cultivares de cebola, nas condições do Submédio do Vale do São Francisco.

\section{MATERIAL E MÉTODOS}

O experimento foi conduzido de maio a novembro de 2011, em Petrolina, PE (99' S, 40²9' 'W, 365.5 m de altitude). Segundo a classificação climática de Köppen, a região apresenta clima do tipo BSWh', semiárido, e valores médios anuais das variáveis climatológicas: temperatura do ar $=26.5^{\circ} \mathrm{C}$, precipitação pluvial $=541.1 \mathrm{~mm}$, umidade relativa do ar $=65,9 \%$, evaporação do tanque classe "A" $=2.500 \mathrm{~mm}$ por ano e velocidade do vento $=$ $2,3 \mathrm{~m} \mathrm{~s}^{-1}$. A precipitação é irregularmente distribuída no espaço e no tempo, concentrando-se nos meses de dezembro a abril; a insolação anual é superior a $3.000 \mathrm{~h}$ (Azevedo et al., 2006). O solo, classificado como Latossolo Vermelho-Amarelo distróferrico, apresentou $\mathrm{pH}\left(\mathrm{H}_{2} \mathrm{O}\right)=6,1 ; \mathrm{Ca}=2,0 \mathrm{cmol}_{\mathrm{c}} \mathrm{dm}^{-3} ; \mathrm{Mg}=0,7 \mathrm{cmol}_{\mathrm{c}} \mathrm{dm}^{-3}$; $\mathrm{Na}=0,01 \mathrm{cmol}_{\mathrm{c}} \mathrm{dm}^{-3} ; \mathrm{K}=0,32 \mathrm{cmol}_{\mathrm{c}} \mathrm{dm}^{-3} ; \mathrm{Al}=0,00 \mathrm{cmol}_{\mathrm{c}}$ $\mathrm{dm}^{-3}, \mathrm{P}($ Mehlich $)=23,8 \mathrm{mg} \mathrm{dm}^{-3}$ e M.O. $=4,1 \mathrm{~g} \mathrm{~kg}^{-1} \mathrm{e}$ físicas $(\%)$ : areia $=83$; silte $=10$ e argila $=7$.

$\mathrm{O}$ delineamento experimental utilizado foi de blocos ao acaso, no esquema fatorial $5 \times 2$, compreendendo cinco doses de fósforo $\left(0 ; 60 ; 120 ; 180\right.$ e $\left.240 \mathrm{~kg} \mathrm{ha}^{-1} \mathrm{de}_{2} \mathrm{O}_{5}\right)$ e dois cultivares tradicionalmente plantados na região (Franciscana IPA-10 e Vale Ouro IPA-11), com quatro repetições. As doses referentes a cada tratamento foram distribuídas e incorporadas manualmente, no sulco de plantio, à profundidade de $3 \mathrm{~cm}$, em cada unidade experimental, tendo como fonte de $\mathrm{P}$ o superfosfato triplo (45\% de $\mathrm{P}_{2} \mathrm{O}_{5}$ ).

A unidade experimental constou de oito linhas de 3,0 m de comprimento, espaçadas de $0,15 \mathrm{~m}$, com $0,10 \mathrm{~m}$ entre plantas, perfazendo uma área total 3,6 $\mathrm{m}^{2}$ (3,0 x 1,2 m), sendo utilizadas como a área útil as seis linhas centrais, retirando-se $0,50 \mathrm{~m}$ em cada extremidade $\left(1,80 \mathrm{~m}^{2}\right)$. A adubação de plantio constou da aplicação de $90 \mathrm{~kg} \mathrm{ha}^{-1}$ de 
$\mathrm{K}_{2} \mathrm{O}$ e $180 \mathrm{~kg} \mathrm{ha}^{-1}$ de $\mathrm{N}$ (Resende et al., 2009). As adubações potássica e nitrogenada foram divididas em três parcelamentos, sendo a primeira realizada no plantio (1/3) e as restantes (2/3) em duas coberturas aos 25 e 50 dias após transplantio, aplicadas em sulcos de plantio a $3 \mathrm{~cm}$ de profundidade. Como fonte de nitrogênio, utilizou-se a ureia, e, de potássio, o cloreto de potássio.

O transplante das mudas ocorreu aos 30 dias após a semeadura, em junho de 2011, e o preparo do solo constou de aração, gradagem e levantamento dos canteiros a $0,20 \mathrm{~m}$ de altura. As irrigações foram feitas por microaspersão, com turno de dois dias e lâminas de água de 10-11 $\mathrm{mm}$, calculada em função da evaporação do tanque classe A, e os tratos fitossanitários foram os comuns à cultura da cebola.

A colheita foi realizada em setembro de 2011, quando as plantas apresentaram sinais avançados de senescência, como amarelecimento e seca das folhas e quando mais de $70 \%$ das plantas encontravam-se estaladas. A cura foi realizada ao sol, por três dias e, à sombra, por 12 dias, em galpão ventilado.

Foram avaliadas a produtividade comercial de bulbos (bulbos perfeitos e com diâmetro transversal acima de 35 $\mathrm{mm}$ ) e a não comercial (refugos), ou seja bulbos com diâmetro inferior a $35 \mathrm{~mm}$ ), expressas em $\mathrm{t} \mathrm{ha}^{-1}$, aos 15 dias após a cura. A massa fresca de bulbo (g/bulbo) foi determinada dividindo-se o peso de bulbos comerciais, após a cura, pelo número de bulbos. A classificação de bulbos comerciais, em percentagem, segundo o diâmetro transversal (mm) em Classe 2: maior que 35 até $50 \mathrm{~mm}$ de diâmetro e Classe 3: maior que 50 até $90 \mathrm{~mm}$. Após o período de cura, os bulbos foram armazenados à temperatura ambiente e realizadas pesagens aos 30 e 60 dias, sendo os valores comparados àqueles obtidos ao final da cura (15 dias após colheita). A temperatura média no período de conservação foi de $27,0{ }^{\circ} \mathrm{C}$, mínima de $21,5^{\circ} \mathrm{C}$, máxima de $33,2{ }^{\circ} \mathrm{C}$ e umidade relativa de $57,7 \%$. Os valores foram transformados em percentagem de perda de peso. Os dados de percentagem foram transformados em arco-seno $\sqrt{\mathrm{P} / 100}$, para efeitos de análise estatística.

Também foi determinada a dose mais econômica de fósforo para a produtividade de bulbos de cebola, conforme Raij (1991) e Natale et al. (2011). Neste estudo, considerou-se o preço médio por kg de bulbo de cebola comercializado no Mercado do Produtor de Juazeiro, no valor de R \$1,24, referente ao ano de 2012 (SEAGRI, 2013). $\mathrm{O}$ custo do $\mathrm{kg}$ de fósforo, cuja fonte foi o superfosfato triplo, no mercado local, que foi $\mathrm{R} \$ 3,33$ (por kg do elemento). Dessa maneira, a "moeda" utilizada nos cálculos, durante todo o estudo, foi a própria cebola, considerando-se a seguinte relação de equivalência: $\mathrm{kg}$ de fósforo aplicado/kg de cebola comercializada igual a $\mathrm{R} \$ 3,33: \mathrm{R} \$ 1,24=2,68$.
A dose econômica foi calculada com base na derivada da equação de regressão entre a produção de bulbos e as doses de fósforo aplicadas, tornando-a igual à relação de troca, ou seja: $d y / d x=a_{1}+2 a_{2} x=$ relação de troca. A dose mais econômica $(x$ ') foi então calculada por:

$x^{\prime}=\mathrm{a}_{1}-\underline{\text { relação de troca }}$

$$
2 .\left(-a_{2}\right)
$$

Os dados coletados foram submetidos à análise de variância e regressão com base no modelo polinomial, utilizando-se o teste $\mathrm{F}$ para comparação dos quadrados médios, a 5\% de probabilidade. Para cultivares, as médias foram comparadas pelo teste de $\mathrm{F}(\mathrm{Gl}=1)$, a $5 \%$ de probabilidade, e as doses de fósforo foram ajustadas a equações de regressão polinomiais, adotando-se como critério para escolha do modelo o efeito significativo pelo teste de F, a $5 \%$ de probabilidade, e a magnitude dos coeficientes de determinação, empregando-se o programa SISVAR 5.0 (Ferreira, 2011).

\section{RESULTADOS E DISCUSSÃO}

Para produtividade comercial, não foi observada diferença significativa entre os cultivares Franciscana IPA-10 (74,6 t ha $\left.{ }^{-1}\right)$ e Vale Ouro IPA-11 (76,1 t ha $\left.{ }^{-1}\right)$, bem como não houve interação entre cultivar e doses de fósforo (Tabela 1). Esses resultados são valores superiores ao da produtividade média nacional $\left(24,7 \mathrm{t} \mathrm{ha}^{-1}\right)$ e aos dos estados de Pernambuco e Bahia, maiores produtores do nordeste, com respectivos 29,0 e 20,4 tha-1 (IBGE, 2013). A dose de 132 $\mathrm{kg} \mathrm{ha}^{-1}$ de $\mathrm{P}_{2} \mathrm{O}_{5}$ promoveu maior produtividade comercial $\left(80,0\right.$ t ha $\left.^{-1}\right)$ (máxima eficiência física do insumo), resultados estes próximos aos encontrados por Yadav \& Prasad (2009), que observaram maior produtividade com a dose de $100 \mathrm{~kg} \mathrm{ha}^{-1}$ de $\mathrm{P}_{2} \mathrm{O}_{5}$ (sem informar o teor do nutriente no solo), como também por Lima et al. (1984) $\left(57 \mathrm{mg} \mathrm{dm}^{-3} \mathrm{de}\right.$ fósforo no solo) e Rizk et al. (2012) (3,4 $\mathrm{mg} \mathrm{dm}^{-3} \mathrm{de}$ fósforo no solo), que obtiveram maior produtividade com as doses de 127,0 e $107,0 \mathrm{~kg} \mathrm{ha}^{-1}$ de $\mathrm{P}_{2} \mathrm{O}_{5}$, respectivamente.

Salienta-se que a área utilizada para o experimento apresentou solo com teor de $\mathrm{P}($ Mehlich $)=23,8 \mathrm{mg} \mathrm{dm}^{-3}, \mathrm{o}$ que é considerado bom e, de acordo com Cavalcanti (2008), a recomendação seria a aplicação de $90 \mathrm{~kg} \mathrm{ha}^{-1} \mathrm{de}_{2} \mathrm{O}_{5}$. Os resultados contrariam em parte esta recomendação, indicando ser a dose mais adequada para as condições deste trabalho a de $130 \mathrm{~kg} \mathrm{ha}^{-1}$ de $\mathrm{P}_{2} \mathrm{O}_{5}$, o que, provavelmente, é justificado pelas maiores produtividades nele alcançadas, tendo em vista que a recomendação leva em consideração produtividade de 40,0 $\mathrm{t} \mathrm{ha}^{-1}$. Analisando-se pela recomendação para Minas Gerais para a cultura (Fontes, 1999), estes valores seriam bem superiores, da ordem de $220 \mathrm{~kg}$ ha-1 de $\mathrm{P}_{2} \mathrm{O}_{5}$, em função de ser o solo classificado como de média disponibilidade. 
Utilizando-se a equação de regressão da Figura 1 calculou-se a dose econômica de fósforo para maior produtividade de bulbos de cebola $(165,40-2,68) /(2 \times 0,628)=$ $162,72 / 1,256=130 \mathrm{~kg} \mathrm{ha}^{-1} \mathrm{de}_{2} \mathrm{O}_{5}$.

A receita prevista, decorrente da adubação, pode ser determinada pelo aumento de produção de bulbos $=10,9$ $\mathrm{t} \mathrm{ha}^{-1}$ (produção de bulbos com a dose mais econômica de P igual a 80,0 t ha-1 menos a produção com a dose zero, igual a $\left.69,1 \mathrm{t} \mathrm{ha}^{-1}\right)$. Subtraindo-se o custo do adubo fosfatado, em kg de bulbo $(130 \mathrm{~kg} \mathrm{x} \mathrm{3,33=432,9/1,24=}$ $349 \mathrm{~kg} \mathrm{ha}^{-1}$ ou $\left.0,349 \mathrm{t} \mathrm{ha}^{-1}\right)$, obteve-se produção de $10,5 \mathrm{t}$ $\mathrm{ha}^{-1}$ de bulbos comerciais, ou seja, uma receita líquida da ordem de $\mathrm{R} \$ 13.020,00\left(10,5 \mathrm{tha}^{-1} \mathrm{x} \mathrm{R} \$ 1,24\right)$.

É importante destacar que a produtividade obtida com a dose econômica ( $130 \mathrm{~kg} \mathrm{ha}^{-1} \mathrm{de}_{2} \mathrm{O}_{5}$ ) esteve muito próxima da produtividade máxima física possível $\left(132 \mathrm{~kg} \mathrm{ha}^{-1}\right.$ de $\mathrm{P}_{2} \mathrm{O}_{5}$ ), o que representa $98,5 \%$.

Resultados similares foram verificados para produção de refugos (bulbos não comerciais). O cultivar Vale Ouro IPA-11 com $0,75 \mathrm{t} \mathrm{ha}^{-1}$ produziu mais refugos que o cultivar Franciscana IPA-10 $\left(0,56 \mathrm{t} \mathrm{ha}^{-1}\right)$ (Tabela 1$)$, resultados semelhantes aos obtidos por Costa et al. (2000). Verificou-se com o aumento das doses uma redução gradativa da produção de bulbos não comerciais, sendo a menor produção estimada a obtida com a dose de $124 \mathrm{~kg} \mathrm{ha}^{-1}$ de $\mathrm{P}_{2} \mathrm{O}_{5}$ (Figura 1). Esses resultados mostram a capacidade de resposta da cebola à aplicação de fósforo e alicerçam as afirmações de diferentes autores, que relatam que o elemento contribui marcadamente para melhor produtividade da cultura, sobretudo, na produção de bulbos de maior tamanho (Singh \& Singh, 2000; Singh et al., 2000; Meena et al., 2007; Yadav \& Prasad, 2009; Sharangi \& Sahu, 2009).

Os resultados positivos da adubação fosfatada, alcançados neste trabalho, devem-se ao papel funcional do fósforo no metabolismo de desenvolvimento da cebola. $\mathrm{O}$ $\mathrm{P}$ é componente estrutural de macromoléculas, como ácidos nucleicos, fosfolipídeos e adenosina trifosfato (ATP). Tem papel fundamental na divisão celular e faz parte da estrutura química de compostos essenciais ao metabolismo vegetal, sendo essencial ao crescimento das partes aérea e radicular das plantas (Malavolta, 2006). Assim, o nutriente favorece o desenvolvimento do sistema radicular das hortaliças, aumentando a absorção de água e de nutrientes; melhorando a qualidade e o rendimento dos produtos colhidos (Moura et al., 2001). Especificamente na cebola, relacionam- se a precocidade, o maior diâmetro dos bulbos e a produtividade (Malavolta, 2006) com o

Tabela 1: Produtividade comercial e refugos (não-comercial) de bulbos, massa fresca, percentagem de perda de massa e classificação em classes de bulbos (\%), segundo o diâmetro transversal de bulbos

\begin{tabular}{lccc}
\hline \multirow{2}{*}{ Variáveis } & \multicolumn{2}{c}{ Cultivares } & \multirow{2}{*}{$\mathbf{C V}^{(\%)}$} \\
\cline { 2 - 3 } & Franciscana IPA-10 & ValeOuro IPA-11 & 8 \\
\hline Produtividade $\left(\mathrm{t} \mathrm{ha}^{-1}\right)$ & $74,6 \mathrm{a}$ & $76,1 \mathrm{a}$ & 18 \\
Refugos $\left(\mathrm{t} \mathrm{ha}^{-1}\right)$ & $0,56 \mathrm{~b}$ & $0,75 \mathrm{a}$ & 6 \\
Massa fresca $\left(\mathrm{g} \mathrm{bulbo}^{-1}\right)$ & $119,9 \mathrm{a}$ & $121,1 \mathrm{a}$ & 8 \\
\% perda massa 30 dias & $12,1 \mathrm{a}$ & $13,1 \mathrm{a}$ & 5 \\
\% perda massa 60 dias & $27,2 \mathrm{~b}$ & $31,9 \mathrm{a}$ & 7 \\
$\%$ bulbos classe 2 & $14,0 \mathrm{a}$ & $8,0 \mathrm{~b}$ & 4 \\
\% bulbos classe 3 & $86,0 \mathrm{~b}$ & $92,0 \mathrm{a}$ & \\
\hline
\end{tabular}

Médias seguidas pela mesma letra, na coluna, não diferem entre si, pelo teste de $\mathrm{F}$ a $5 \%$ de probabilidade.

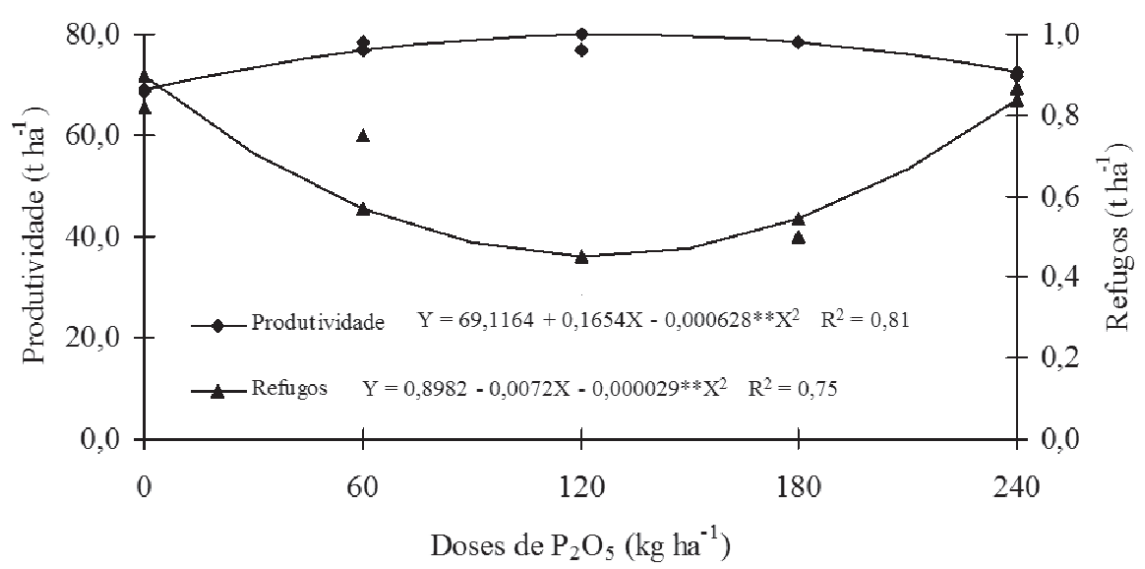

Figura 1: Produtividade comercial e de refugos (não comercial) de bulbos de cultivares de cebola, em função de doses de fósforo.

Rev. Ceres, Viçosa, v. 63, n.2, p. 249-255, mar/abr, 2016 
suprimento de fósforo, que favorece um amplo desenvolvimento do sistema radicular, aumentando a absorção de água e nutrientes (Filgueira, 2008).

Também não se constataram efeitos significativos da interação cultivar e doses de fósforo para massa fresca de bulbo. O cultivar Franciscana (119,9 g/bulbo) não mostrou diferenças quanto ao cultivar ValeOuro IPA-11 (121,1 $\mathrm{g} / \mathrm{bulbo}$ ). Bulbos com maior massa fresca foram obtidos com a dose de $131 \mathrm{~kg} \mathrm{ha}^{-1}$ de $\mathrm{P}_{2} \mathrm{O}_{5}$ (figura 2). Singh et al. (2000) e Faheema et al. (2009) obtiveram melhores massas frescas de bulbos com 75 e $80 \mathrm{~kg} \mathrm{ha}^{-1} \mathrm{de}_{2} \mathrm{O}_{5}$, respectivamente.

A conservação pós-colheita apresentou resultados diferenciados de doses de fósforo e cultivares, em função do período de armazenagem. Para perda de massa aos 30 dias após cura, não se constataram efeitos significativos entre cultivares e doses de fósforo. O cultivar Franciscana IPA-10 apresentou perda da ordem de $12,1 \%$ e, o cultivar Vale Ouro IPA-11, de 13,1\% (Tabela 1). Para doses, obteve-se variação entre 10,1 e 13,1\%. No que se refere à perda de massa fresca dos bulbos, aos 60 dias após cura, detectou-se efeito significativo apenas para cultivares, com menor perda para o cultivar Franciscana IPA-10 (27,2\%), em comparação com a do cultivar Vale Ouro IPA$11(31,9 \%)$ (Tabela 1). Para doses, observaram-se variações de 27,4 a 30,9\%. Morsy et al. (2012) verificaram que as doses de $71 \mathrm{~kg} \mathrm{ha}^{-1}$ de $\mathrm{P}_{2} \mathrm{O}_{5}$ mais $114 \mathrm{~kg} \mathrm{ha}^{-1}$ de $\mathrm{K}_{2} \mathrm{O}$ fizeram decrescer significativamente a perda de massa fresca dos bulbos, após seis meses de armazenamento. Maior conservação pós-colheita tem relevante importância por ocasião da comercialização do produto, que apresenta grandes variações de preços, em curtos períodos de tempo. O produtor, em função do conhecimento das perdas de massa da cebola, poderá alcançar melhores cotações de preços e incrementar ou maximizar seus lucros, em função da volatilidade do mercado ceboleiro. As menores perdas de massa observadas para o cultivar Franciscana IPA-10 podem estar relacionadas com o maior teor de só- lidos solúveis. Bandeira et al. (2013) informam valores significativamente superiores para o cultivar Franciscana IPA-10 (11,72\%), em comparação com as do cultivar ValeOuro IPA-11 (10,81\%). Segundo Chagas et al. (2004), maior teor de sólidos solúveis está ligado à alta pungência e à boa qualidade de armazenamento dos bulbos.

Para classificação de bulbos comerciais de cebola registraram-se efeitos independentes dos tratamentos, para as diferentes classes. No que se refere à classificação de bulbos classe 2, que são os de tamanho inferior (maior que 35 até $50 \mathrm{~mm}$ de diâmetro), o cultivar Franciscana IPA-10 apresentou maior percentagem de bulbos $(14,0 \%)$ que o cultivar Vale Ouro IPA-11 (8,0\%). Com relação à classe 3, que são bulbos de maior calibre (maior que 50 até 90 mm de diâmetro) os resultados demonstraram maior proporção nessa classe para o cultivar Vale Ouro IPA-11 $(92,0 \%)$ (Tabela 1). A obtenção de bulbos maiores, além de estar diretamente relacionada com o aumento do rendimento e com as características genéticas do cultivar, também aumenta a lucratividade, pois bulbos com diâmetro inferior a $50 \mathrm{~mm}$ apresentam menor valor de mercado do que bulbos de diâmetros superiores (Kurtz et al., 2012).

Com relação à aplicação de fósforo (figura 3), observaram-se, para a classe 2, redução linear com o incremento das doses de $\mathrm{P}$, sendo este valor da ordem de $1,36 \%$ a cada incremento da dose. A dose $240 \mathrm{~kg} \mathrm{ha}^{-1}$ de $\mathrm{P}_{2} \mathrm{O}_{5}$ propiciou a menor produção de bulbos nessa classe $(17,0 \%)$, ou seja, doses menores aumentaram proporcionalmente a produção de bulbos pequenos. Com relação à classe 3 , que são bulbos maiores, verificou-se relação inversa, ou seja, um incremento linear com o aumento das doses de P, constatando-se, a cada aumento da dose, $1,8 \%$ na produção de bulbos nessa classe, retratando, dessa forma, que esse nutriente além de contribuir para a melhoria da produtividade, também contribui para a produção de bulbos de maior diâmetro. Singh et al. (2000), Singh \& Singh (2000) e Faheema et al. (2009), também obtiveram bulbos de maior diâmetro com o aumento das doses de fósforo.

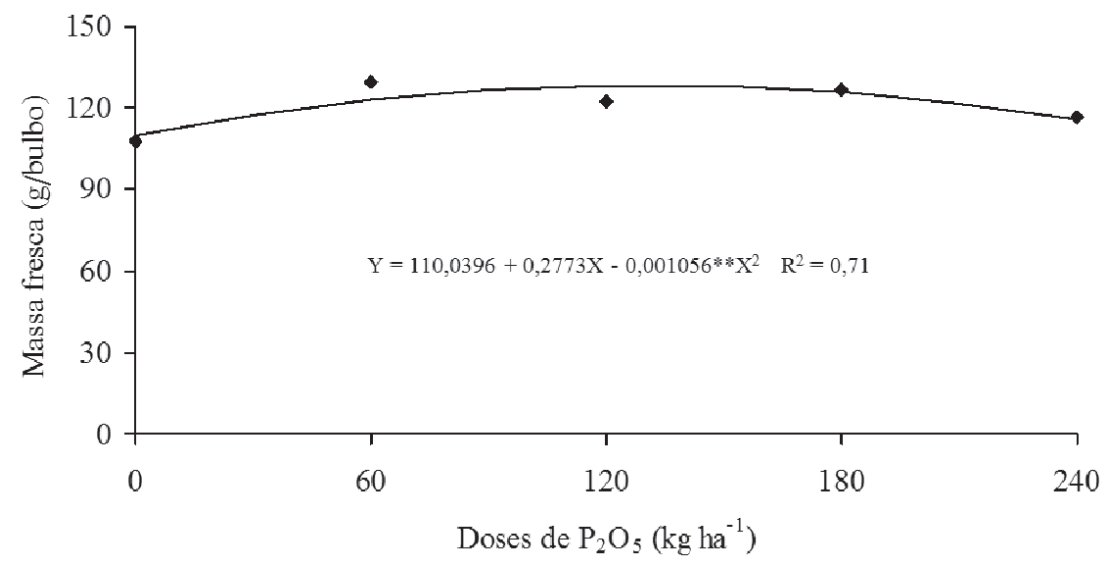

Figura 2: Massa fresca de bulbos de cultivares de cebola, em função de doses de fósforo. 


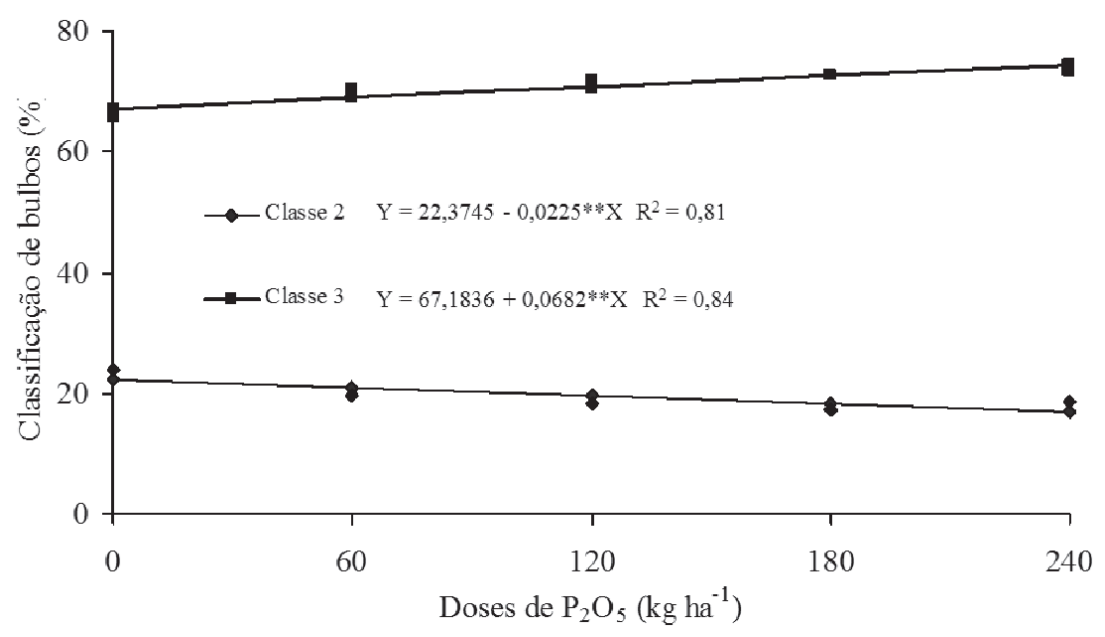

Figura 3: Classificação de bulbos em classes (\%), segundo o diâmetro transversal de bulbos de cebola, em função de doses de fósforo.

\section{CONCLUSÕES}

A dose de $130 \mathrm{~kg} \mathrm{ha}^{-1}$ de $\mathrm{P}_{2} \mathrm{O}_{5}$, independentemente do cultivar, proporcionou maior produtividade de bulbos comerciais.

Na conservação pós-colheita dos bulbos, aos 30 dias após cura, não se verificaram perdas significativas de massa dos bulbos para cultivares ou doses de fósforo.

Aos 60 dias após cura, melhor conservação pós-colheita foi apresentada pelo cultivar Franciscana IPA-10, em comparação com a do cultivar Vale Ouro.

\section{REFERÊNCIAS}

Azevedo PV, Sousa IF, Silva BB \& Silva VPR (2006) Water-use efficiency of dwarf-green coconut (Cocos nucifera L.) orchards in Northeast Brazil. Agricultural Water Management, 1:259-264.

Balemi T \& Negisho K (2012) Management of soil phosphorus and plant adaptation mechanisms to phosphorus stress for sustainable crop production: a review. Journal of Soil Science and Plant Nutrition, 12:547-561.

Bandeira GRL, Queiroz SOP, Aragão CA, Costa ND \& Santos CAF (2013) Desempenho agronômico de cultivares de cebola sob diferentes manejos de irrigação no Submédio São Francisco. Irriga, 18:73-84.

Bruulsema T, Lemunyon J \& Herz B (2009) Fundamentos para utilização correta do seu fertilizante. Informações Agronômicas, $126: 15-18$

Cavalcanti FJ de A (2008) Recomendações de adubação para o Estado de Pernambuco: $2^{\mathrm{a}}$ aproximação. $3^{\mathrm{a}}$ ed. Recife, IPA. 212p.

Chagas SJR, Resende GM \& Pereira LV (2004) Características qualitativas de cultivares de cebola no sul de Minas Gerais. Ciência e Agrotecnologia, 28:102-106.

Costa ND, Resende GM \& Dias RCS (2000) Avaliação de cultivares de cebola em Petrolina-PE. Horticultura Brasileira, 18:57-60.

Coutinho ELM, Natale W \& Souza ECA (1993) Adubos e corretivos: aspectos particulares na olericultura. In: Ferreira ME, Castellane PD \& Cruz MCP (Eds.) Nutrição e adubação de hortaliças. Piracicaba, Potafos. p.85-140.
Dudhat MS, Chovatia PK, Sheta BT, Rank HD \& Parmar HV (2010) Effect of nitrogen, phosphorous and potash on growth and bulb yield of onion (Allium cepa L.). Asian Journal of Soil Science, 5:189-191.

Faheema S, Ahmed N, Hussain K, Narayan S \& Chattoo MA (2009) Response of long day onion cv. Yellow Globe to different levels of nitrogen phosphorus and potassium under temperate conditions of Kashmir Valley. Asian Journal of Horticulture, 4:131-133.

Ferreira DF (2011). Sisvar: a computer statistic alanalysis system. Ciência e Agrotecnologia, 35:1039-1042.

Filgueira FAR (2008) Novo manual de olericultura: agrotecnologia moderna na produção e comercialização de hortaliças. $3^{\mathrm{a}}$ ed. Viçosa, Editora UFV. 421p.

Fixen PE (2009) Reservas mundiais de nutrientes dos fertilizantes. Informações Agronômicas, 126:8-14.

Fontes PCR (1999) Sugestões de adubação para hortaliças. In: Ribeiro AC, Guimarães PTG \& Alvarez VVH (Eds.) Recomendações para o uso de corretivos e fertilizantes em Minas Gerais $5^{\text {a }}$ Aproximação. Viçosa, CFSEMG. p.171-208.

Grant CA, Platen DN, Tomaziewicz DJ \& Sheppard SC (2001) A importância do fósforo no desenvolvimento inicial da planta. Informações agronômicas, 95:26-30.

IBGE - Instituto Brasileiro de Geografia e Estatística (2013) Levantamento Sistemático da Produção Agrícola. Rio de Janeiro, IBGE. p.1-84.

Kurtz C, Ernani PR, Coimbra JLM \& Petry E (2012). Rendimento e conservação de cebola alterados pela dose e parcelamento de nitrogênio em cobertura. Revista Brasileira de Ciência do Solo, 36:865-876.

Malavolta E (2006) Manual de nutrição de plantas. São Paulo, Agronômica Ceres. 638p.

Meena PM, Kumar V, Meena VS \& Mohit M (2007) Effect of phosphorus doses on growth and yield of onion (Allium cepa L.) cv. Nasik red. Agricultural Science Digest, 27:301-302.

Morsy MG, Marey RA, Karam SS \& Abo-Dahab AMA (2012) Productivity and storability of onion as influenced by the different levels of NPK fertilization. Journal Agricultural Research Kafr El-Sheikh University, 38:171-187. 
Moura WM, Lima PC, Casali VWD \& Pereira PRG (2001) Eficiência nutricional para fósforo em linhagens de pimentão. Horticultura Brasileira, 19:306-312.

Natale W, Rozane DE, Prado RM, Romualdo LM, Souza HAS \& Hernandes A (2011) Dose econômica de calcário na produtividade de caramboleiras. Revista Brasileira de Fruticultura, 33:1294-1299.

Lima JA, Buso JA, Souza AF, Makishima N, Junqueira JGO, Ferreira PF \& C Filho J (1984) Produção de cebola em função de níveis de aplicação de nitrogênio e fósforo. Horticultura Brasileira, 2:12-14.

Porto DR de Q, Cecílio Filho AB, May A \& Vargas PF (2007) Acúmulo de macronutrientes pela cultivar de cebola "Superex" estabelecida por semeadura direta. Ciência Rural, 37:949-955.

Raij B van (1991) Fertilidade do solo e adubação. Campinas, Ceres. $343 p$.

Resende GM, Costa ND \& Pinto JM (2009) Rendimento e conservação pós-colheita de bulbos de cebola com doses de nitrogênio e potássio. Horticultura Brasileira, 27:7-11.

Resende LM de A, Mascarenhas MHT \& Simão MLR (2002) Panorama da produção e da comercialização da cebola em Minas Gerais. Informe Agropecuário, 23:7-19.

Rizk FA, Shaheen AM, Abd El-Samad EH \& Sawan OM (2012) Effect of different nitrogen plus phosphorus and sulphur fertilizer levels on growth, yield and quality of onion (AIlium cepa L.). Journal of Applied Sciences Research, 8:3353-3361.

SEAGRI - Secretaria de Agricultura, Irrigação e Reforma Agrária do Estado da Bahia (2013) Cotação Agrícola. Disponível em: <http://www.seagri.ba.gov.br/cotacao.asp>. Acessado em: $27 \mathrm{de}$ maio de 2013.

Sharangi AB \& Sahu PK (2009) Effect of Placement and Dose of phosphatic fertilizers on onion. Journal of Plant Nutrition, 32:1901-1913
Singh JV, Kumar A \& Singh C (2000) Influence of phosphorus on growth and yield of onion (Allium cepa L.). Indian Journal Agricultural Research, 34:51-54.

Singh RB \& Singh SB (2000) Significance of nitrogen, phosphorus and potassium on onion (Allium cepa L.) raised from onion sets (bulblets). Vegetable Science, 27:88-89.

Tawaraya K, Hirose R \& Wagatsuma T (2012) Inoculation of arbuscular mycorrhizal fungi can substantially reduce phosphate fertilizer application to Allium fistulosum L. and achieve marketable yield under field condition. Biology and Fertility of Soils, 18:839-843.

Tekalign T, Abdissa Y \& Pant LM (2012) Growth, bulb yield and quality of onion (Allium cepa L.) as influenced by nitrogen and phosphorus fertilization on vertisol. II: Bulb quality and storability. African Journal of Agricultural Research, 7:58805985.

Vance CP, Uhde-Stone C \& Allan D (2003) Phosphorus acquisition and use: critical adaptation by plants for securing non-renewable resources. New Phytologist, 157:423-447.

Vidigal SM, Moreira MA \& Pereira PRG (2010) Crescimento e absorção de nutrientes pela planta cebola cultivada no verão por semeadura direta e por transplantio de mudas. Bioscience Journal, 26:59-70.

Wamser AF, Suzuki A, Mueller S, Gonçalves M de M, Valmorbida J \& Becker WF (2011) Adubação fosfatada e potássica da cebola em área com resíduos de adubação do tomateiro. Agropecuária Catarinense, 24:86-88.

Yadav D \& Prasad VM (2009) Response of kharif onion (Allium cepa L.) to nitrogen, phosphorus and zinc application. Plant Archives, 9:37-39. 